\title{
An Epidemiological Characteristic of the COVID-19 Among Children
}

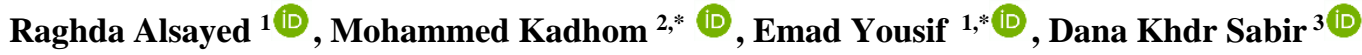 \\ 1 Department of Chemistry, College of Science, Al-Nahrain University, Baghdad, Iraq \\ 2 Department of Environment, College of Energy and Environmental Sciences, Alkarkh University of Science, Baghdad, \\ Iraq \\ 3 Department of Medical Laboratory Sciences, Charmo University, Sulaimani, Kurdistan Region, Iraq \\ * Correspondence: kadhom@kus.edu.iq (M.K); emad_yousif@hotmail.com (E.Y.);
}

Scopus Author ID 26533612800

Received: 25.05.2020; Revised: 11.06.2020; Accepted: 12.06.2020; Published: 15.06.2020

\begin{abstract}
A novel Coronavirus disease 2019 (COVID-19) is an infectious disease caused by SARS coronavirus 2 (SARS-CoV-2), which affects the respiratory system of human beings. Until now, numbers of COVID-19 cases have been reported among children; in China, less than $2.4 \%$ of the total cases occurred in those aged under 19 years old. SARS-CoV-2 transmission in children mostly occurs through contact with adult patients, primarily through exposure in the home. This article discusses the epidemiological features of COVID-19 in children.
\end{abstract}

Keywords: COVID-19; adolescent; ACE2; infection; children; SARS-CoV-2.

(C) 2020 by the authors. This article is an open-access article distributed under the terms and conditions of the Creative Commons Attribution (CC BY) license (https://creativecommons.org/licenses/by/4.0/).

\section{Introduction}

Among the first cases in Wuhan/China, the place where COVID-19 was spread and detected, there were no children involved, which suggested that children are not symptomatic of the disease [1-8]. Now on Jun $13^{\text {th }} 27^{\text {th }}, 2020$, the disease is pandemic with nearly 7.8 million confirmed cases and 430 thousand deaths, including around $2 \%$ confirmed cases amoung children. Children have been described as being under the age of 18 years old. Results reported preliminary from china, and these recently reported from Europe and the United States show that elderly people, in particular, those with underlying health conditions, are at a higher risk of serious COVID-19- accompanied diseases and death comparing to children [9-15]. In the Chinese Center for Disease Control and Prevention's (China CDC) first major study, where 44,672 confirmed COVID-19 cases were covered, only one case of death recorded in an individual of 19 years old, while nearly $80 \%$ of the total deaths took place within elderly people of the age $\geq 60$ years [16-27]. However, no children under the age of 10 died except a 1-year old child who had a serious form of the disease. Similarly, for patents of age between 10-19 years, one death was reported [28-37]. In the United States, the age distribution relationship with cases ratios was shown in Figure 1 [38-45]:

There are very few pregnancies and neonatal studies on COVID-19 to clarify whether or not babies can become infected in utero. A case series of 31 COVID-19 pregnant women showed none of their babies or placenta positively screened for the virus [46-51]. There is one case of a newborn baby who was born by cesarean section with a positive SARS-CoV-2. Swab 
(PCR) test was used 16 hours after birth with no detectable maternal SARS-CoV-2 antibodies until after delivery, indicating a possible vertical transmission [52-56].

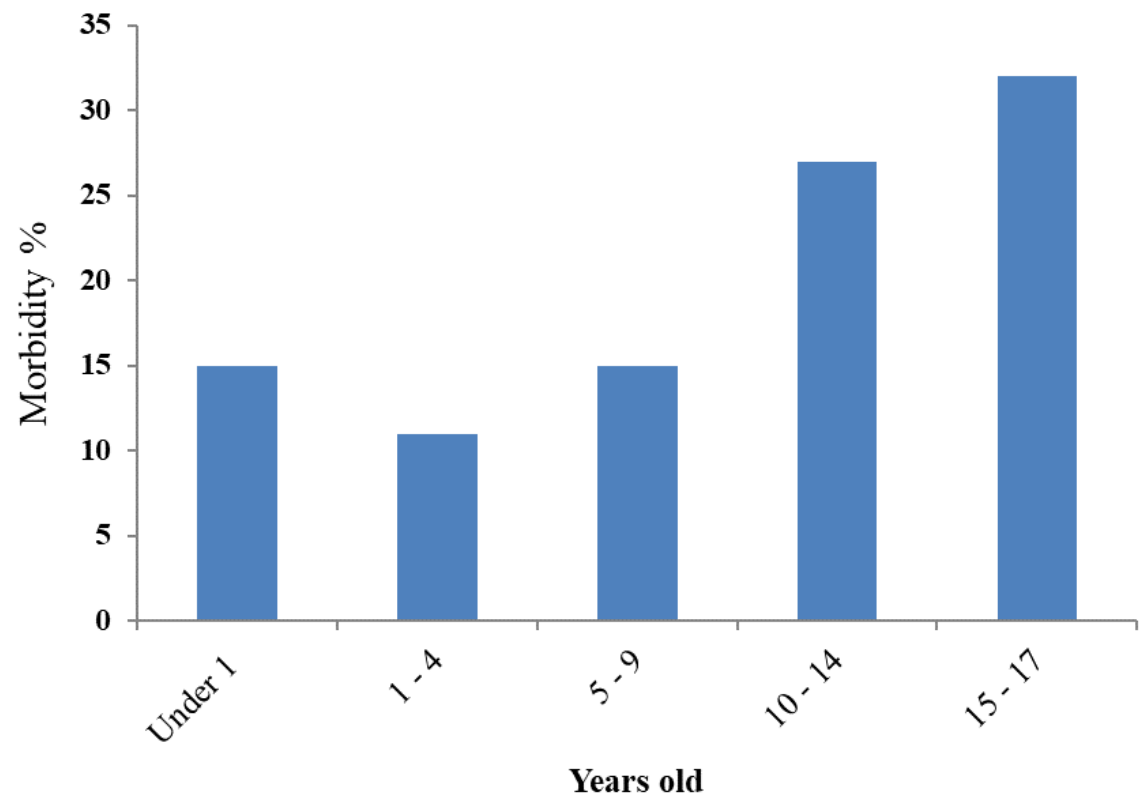

Figure 1. Age distribution among COVID-19 patients aged less than 18 years old in the United States

\section{Symptoms}

COVID-19 has different symptoms that could differ from a person to another. However, the most common symptoms and signs in children were cough, follow by pharyngeal erythema, and fever. Table 1 shows the percentage of the common COVID-19 symptoms that appear among children [57-63].

Table 1. Common COVID-19 symptoms appearance in children.

\begin{tabular}{l|c} 
Symptoms & Appearance ratio (\%) \\
\hline Cough & $48.5 \%$ \\
\hline Pharyngeal erythema & $46.2 \%$ \\
\hline Fever & $41.5 \%$ \\
\hline Tachypnea & $28.7 \%$ \\
\hline Diarrhea, rhinorrhea, and nasal congestion & $10 \%$
\end{tabular}

The ratio of children identified with critical and/or severe conditions of COVID-19 was significantly lower than those of adults with values of $0.6 \%$ and $2.5 \%$, respectively. Children with COVID-19 who had asymptomatic cases were more than children with influenza infection, while pneumonia was more prevalent [64-66].

\section{Epidemiology in children}

The main reason to have a lower incident of COVID-19 cases among children (agreeing with earlier Middle East respiratory syndrome [MERS] and SARS coronaviruses) is remaining elusive. In comparison with older age groups, many theories have been suggested to understand this phenomenon, such as various behaviors of immunological responses over the age ranges. Adults are possibly developing unbalanced immune responses when they get infected with COVID-19, leading to cytokine storms that frequently cause lungs damage and low prognosis for adult patients compared to children and infants. A variation in innate immunity within the different age categories and more successful response of $\mathrm{T}$ cells could be found in children to prevent lung harm [67-70]. 
Cross-protection is obtained from previous contact with human coronavirus ( $\mathrm{HuCoV}$ ), which is commonly accompanied by mild infections within children. Thereby, it was considered as a possible comprehend for lower risk in babies, though it does not seem to be a plausible theory because of the serious cases recorded for very young infants. This age group is improbably exposed to these $\mathrm{HuCoV}$ contagions before [71-78]. Another possibility is that the presence of other simultaneous viruses in the mucosa of lungs and airways, which are common in young children, could limit the growth of SARS-CoV2 by direct virus-to-virus interactions and competition [68].

Recently the role of measles, mumps, and rubella (MMR) vaccination has also been proposed in the children protections against COVID-19. The authors suggested that a broad neutralizing antibody can be produced as the result of MMR vaccine which can protect children from a number of diseases including COVID-19. Such hypothesis was based on short amino acid sequence similarities between Spike (S) glycoprotein of SARS-COV-2 with both Fusion (F1) glycoprotein of Measles virus and envelope (E1) glycoprotein of Rubella virus [79-84].

The highly reasonable hypothesis for low COVID-19 infection among children have been also linked to the production of angiotensin-converting enzyme 2 (ACE2) on alveolar epithelial cells of type I and II. ACE2 was discovered to be SARS-CoV-2 receptor, necessary for entry of host cells and subsequent viral replication [85-89]. Consequently, a restricted existence of ACE2 in childhood, a period of age where lungs are still developing, could prevent the children from serious cases of illness. It is worth mentioning that men tend to have more ACE2 compared to women in their alveolar cells, this may also explain the reason of higher infection rate among males comparing to females [90-94]. This pattern was also noticed in children's research, by comparing the hospitalization infection rates among boys and girls [64]. In addition, children frequently undergo winter respiratory infections (e.g. respiratory syncytial virus (RSV)) and may have a higher number of antibodies to the virus than adults [95-98]. Also, the immunity system of children has still been going to develop and can respond to pathogens differently for adults.

\section{Conclusions}

COVID-19 is found to cause low-risk infections in children comparing with older people for unknown reasons. However, for those who got infected, it is still unclear if the chronic diseases have affected the outcome. In addition, children with asymptomatic or mildly symptomatic could be a carrier to spread the disease to other people such as parents and relatives. Even though, no transmitting of the COVID-19 has been identified to date from children to adults. Children and their families could take action to prevent respiratory infections from spreading [99-103]. To reduce the chances of infection, the following points should be considered:

- Washing hands with soap and water more often for at least 20 seconds, it is the most effective method to prevent infection.

- Alcohol-based disinfection wipes should be used for hand cleaning and disinfection.

- Proper hand hygiene is recommended after coughing or sneezing.

\section{Funding}

This research received no external funding. 


\section{Acknowledgments}

The authors like to thank their affiliation universities for partially supporting this work, namely: Al-Nahrain University, Alkarkh University of Science, and Charmo University.

\section{Conflicts of Interest}

The authors declare no conflict of interest.

\section{References}

1. Li, Q.; Guan, X.; Wu, P.; Wang, X.; Zhou, L.; Tong, Y.; Ren, R.; Leung, K.S.M.; Lau, E.H.Y.; Wong, J.Y.; Xing, X.; Xiang, N.; Wu, Y.; Li, C.; Chen, Q.; Li, D.; Liu, T.; Zhao, J.; Liu, M.; Tu, W.; Chen, C.; Jin, L.; Yang, R.; Wang, Q.; Zhou, S.; Wang, R.; Liu, H.; Luo, Y.; Liu, Y.; Shao, G.; Li, H.; Tao, Z.; Yang, Y.; Deng, Z.; Liu, B.; Ma, Z.; Zhang, Y.; Shi, G.; Lam, T.T.Y.; Wu, J.T.; Gao, G.F.; Cowling, B.J.; Yang, B.; Leung, G.M.; Feng, Z. Early Transmission Dynamics in Wuhan, China, of Novel Coronavirus-Infected Pneumonia. New England Journal of Medicine 2020, 382, 1199-1207, https://doi.org/10.1056/NEJMoa2001316.

2. Roy, A.; Kucukural, A.; Zhang, Y. I-TASSER: a unified platform for automated protein structure and function prediction. Nature Protocols 2010, 5, 725-738, https://doi.org/10.1038/nprot.2010.5.

3. Molecular Operating Environment (MOE), 2019.01; Chemical Computing Group ULC, 1010 Sherbrooke St. West, Suite \#910, Montreal, QC, Canada, H3A 2R7, 2019.

4. Santos, Y.M.; Barraza, S.J.; Wilson, M.W.; Agius, M.P.; Mielech, A.M.; Davis, N.M.; Baker, S.C.; Larsen, S.D.; Mesecar, A.D. X-ray Structural and Biological Evaluation of a Series of Potent and Highly Selective Inhibitors of Human Coronavirus Papain-like Proteases. J.Med.Chem. 2014, 57, 2393-2412, https://doi.org/10.1021/jm401712t.

5. Hilgenfeld, R. From SARS to MERS: crystallographic studies on coronaviral proteases enable antiviral drug design. FEBS J. 2014, 281,4085-4096, https://doi.org/10.1111/febs.12936.

6. Peersen, O.B. Picornaviral polymerase structure, function, and fidelity modulation. Virus Res. 2017, 234, 420, https://doi.org/10.1016/j.virusres.2017.01.026.

7. Báez-Santos, Y.M.; Barraza, S.J.; Wilson, M.W.; Agius, M.P.; Mielech, A.M.; Davis, N.M.; Baker, S.C.; Larsen, S.D.; Mesecar, A.D. X-ray Structural and Biological Evaluation of a Series of Potent and Highly Selective Inhibitors of Human Coronavirus Papain-like Proteases. Journal of Medicinal Chemistry 2014, 57, 2393-2412, http://dx.doi.org/10.1021/jm401712t.

8. Woo, P.C.Y.; Lau, S.K.P.; Lam, C.S.F.; Lau, C.C.Y.; Tsang, A.K.L.; Lau, J.H.N.; Bai, R.; Teng, J.L.L.; Tsang, C.C.C.; Wang, M.; Zheng, B.J.; Chan, K.H.; Yuen, K.Y. Discovery of seven novel Mammalian and avian coronaviruses in the genus deltacoronavirus supports bat coronaviruses as the gene source of alphacoronavirus and betacoronavirus and avian coronaviruses as the gene source of gammacoronavirus and deltacoronavirus. J Virol 2012, 86, 3995-4008, https://doi.org/10.1128/JVI.06540-11.

9. King, A.; Simonetti, J.; Bennett, E.; Simeona, C.; Stanek, L.; Roxby, A.C.; Rowhani-Rahbar, A. Firearm storage practices in households with children: A survey of community-based firearm safety event participants. Preventive Medicine 2020, 131, https://doi.org/10.1016/j.ypmed.2019.105952.

10. Mayer, K.; Nellessen, C.; Hahn-Ast, C.; Schumacher, M.; Pietzonka, S.; Eis-Hubinger, A.M.; Drosten, C.; Brossart, P.; Wolf, D. Fatal outcome of human coronavirus NL63 infection despite successful viral elimination by IFN-alpha in a patient with newly diagnosed ALL. Eur J Haematol 2016, 97, 208-210, https://doi.org/10.1111/ejh.12744.

11. Al-Khannaq, M.N.; Ng, K.T.; Oong, X.Y.; Pang, Y.K.; Takebe, Y.; Chook, J.B.; Hanafi, N.S.; Kamarulzaman, A.; Tee, K.K. Molecular epidemiology and evolutionary histories of human coronavirus OC43 and HKU1 among patients with upper respiratory tract infections in Kuala Lumpur, Malaysia. Virol J 2016, 13, 33, https://doi.org/10.1186/s12985-016-0488-4.

12. Cui, J.; Li, F.; Shi, Z.L. Origin and evolution of pathogenic coronaviruses. Nat. Rev. Microbiol. 2019, 17, 181-192, https://doi.org/10.1038/s41579-018-0118-9.

13. Oong, X.Y.; Ng, K.T.; Takebe, Y.; Ng, L.J.; Chan, K.G.; Chook, J.B.; Kamarulzaman, A.; Tee, K.K. Identification and evolutionary dynamics of two novel human coronavirus OC43 genotypes associated with acute respiratory infections: Phylogenetic, spatiotemporal and transmission network analyses. Emerg. Microbes Infect. 2017, 6, https://doi.org/10.1038/emi.2016.132.

14. Lau, S.K.; Lee, P.; Tsang, A.K.; Yip, C.C.; Tse, H.; Lee, R.A.; So, L.Y.; Lau, Y.L.; Chan, K.H.; Woo, P.C.; Yuen, K.Y. Molecular epidemiology of human coronavirus OC43 reveals evolution of different genotypes over time and recent emergence of a novel genotype due to natural recombination. $J$ Virol 2011, 85, 1132511337, https://doi.org/10.1128/jvi.05512-11. 
15. Menachery, V.D.; Yount, B.L., Jr.; Sims, A.C.; Debbink, K.; Agnihothram, S.S.; Gralinski, L.E.; Graham, R.L.; Scobey, T.; Plante, J.A.; Royal, S.R.; Swanstrom, J.; Sheahan, T.P.; Pickles, R.J.; Corti, D.; Randell, S.H.; Lanzavecchia, A.; Marasco, W.A.; Baric, R.S. SARS-like WIV1-CoV poised for human emergence. Proc Natl Acad Sci U S A 2016, 113, 3048-3053, https://doi.org/10.1073/pnas.1517719113.

16. Liu, Z; Bing, X.; Zhi, X. The Epidemiological Characteristics of an Outbreak of 2019 Novel Coronavirus Diseases (COVID-19) in China. Chin Jour Epidem 2020, 41, 145-151, https://doi.org/10.46234/ccdcw2020.032.

17. Yang, Y.; Liu, C.; Du, L. Y.; Jiang, S.; Shi, Z.; Baric, R.S.; Li, F. Two mutations were critical for bat-tohuman transmission of Middle East Respiratory Syndrome coronavirus. J. Virol. 2015, 89, 9119-9123, https://doi.org/10.1128/JVI.01279-15.

18. De Haan, C.A.; Rottier, P.J. Molecular interactions in the assembly of coronaviruses. Adv Virus Res. 2005, 64, 165-230, https://doi.org/10.1016/S0065-3527(05)64006-7.

19. Masters, P.S. The molecular biology of coronaviruses. Adv Virus Res. 2006, 66, 193-292, https://doi.org/10.1016/S0065-3527(06)66005-3.

20. Raamsman, M.; Locker, J.; Hooge, A.; De Vries, A.; Griffiths, G.; Vennema, H.; Rottier, P. Characterization of the Coronavirus Mouse Hepatitis Virus Strain A59 Small Membrane Protein E. J Virol 2000, 74, 23332342, https://doi.org/10.1128/JVI.74.5.2333-2342.2000.

21. Blau, D.M.; Holmes, K.V. Human coronavirus HCoV-229E enters susceptible cells via the endocytic pathway. Adv Exp Med Biol. 2001, 494, 193-8, https://doi.org/10.1007/978-1-4615-1325-4_31.

22. Chu, V.C.; McElroy, L.J.; Ferguson, A.D.; Bauman, B.E.; Whittaker, G.R. Avian infectious bronchitis virus enters cells via the endocytic pathway. Adv Exp Med Biol. 2006, 581, 309-312, https://doi.org/10.1007/9780-387-33012-9_54.

23. Eifart, P.; Ludwig, K.; Bottcher, C.; de Haan, C.A.; Rottier, P.J.; Korte, T.; Herrmann, A. Role of endocytosis and low $\mathrm{pH}$ in murine hepatitis virus strain A59 cell entry. J Virol 2007, 81, 10758-10768, https://doi.org/10.1128/jvi.00725-07.

24. Graham, R.L.; Baric, R.S. Recombination, reservoirs, and the modular spike: mechanisms of coronavirus cross-species transmission. J Virol. 2010, 84, 3134-3146, https://doi.org/10.1128/JVI.01394-09.

25. Bárcena, M.; Oostergetel, G.T.; Bartelink, W.; Faas, F.G.A.; Verkleij, A.; Rottier, P.J.M.; Koster, A.J.; Bosch, B.J. Cryo-electron tomography of mouse hepatitis virus: Insights into the structure of the coronavirion. Proceedings of the National Academy of Sciences 2009, 106, 582, https://doi.org/10.1073/pnas.0805270106.

26. Lewicki, D.N.; Gallagher, T.M. Quaternary structure of coronavirus spikes in complex with carcinoembryonic antigen- related cell adhesion molecule cellular receptors. J Biol Chem. 2002, 277, 19727 19734, https://doi.org/10.1074/jbc.M201837200.

27. de Haan, C.A.M.; de Wit, M.; Kuo, L.; Montalto-Morrison, C.; Haagmans, B.L.; Weiss, S.R.; Masters, P.S.; Rottier, P.J.M. The glycosylation status of the murine hepatitis coronavirus $M$ protein affects the interferogenic capacity of the virus in vitro and its ability to replicate in the liver but not the brain. Virology 2003, 312, 395-406, https://doi.org/10.1016/S0042-6822(03)00235-6.

28. Wu, Z; McGoogan, J. Characteristics of and important lessons from the coronavirus disease 2019 (COVID19) outbreak in China: summary of a report of 72314 cases from the Chinese Center for Disease Control and Prevention. JAMA 2020, 1239-1242, https://doi.org/10.1001/jama.2020.2648.

29. Ng, M.L.; Tan, S.H., See, E.E.; Ooi, E.E.; Ling, A.E. Proliferative growth of SARS coronavirus in Vero E6 cells. J Gen Virol. 2003, 84, 3291-3303, https://doi.org/10.1099/vir.0.19505-0.

30. Kuo, L.; Masters, P.S. Genetic evidence for a structural interaction between the carboxy termini of the membrane and nucleocapsid proteins of mouse hepatitis virus. J Virol 2002, 76, 4987-4999, https://doi.org/10.1128/jvi.76.10.4987-4999.2002.

31. Keane, S.C.; Giedroc, D.P. Solution of Mouse Hepatit Virus (MHV) nsp3a and determinants of the interaction with MHV nucleocapsid (N) protein. J Virol. 2013, 87, 3502-3515, https://dx.doi.org/10.1128\%2FJVI.03112-12.

32. Weiss, S.R.; Navas-Martin, S. Coronavirus pathogenesis and the emerging pathogen severe acute respiratory syndrome coronavirus. Microbiol Mol Biol Rev 2005, 69, 635-664, https://doi.org/10.1128/MMBR.69.4.635-664.2005.

33. Goldsmith, C.S.; Tatti, K.M.; Ksiazek, T.G.; Rollin, P.E.; Comer, J.A.; Lee, W.W.; Rota, P.A.; Bankamp, B.; Bellini, W.J.; Zaki, S.R. Ultrastructural characterization of SARS coronavirus. Emerg Infect Dis 2004, 10, 320-326, https://doi.org/10.3201/eid1002.030913.

34. Gosert, R.; Kanjanahaluethai, A.; Egger, D.; Bienz, K.; Baker, S.C. RNA replication of mouse hepatitis virus takes place at double-membrane vesicles. J Virol 2002, 76, 3697-3708, https://doi.org/10.1128/jvi.76.8.3697-3708.2002.

35. Lu, R.; Zhao, X.; Li, J.; Niu, P.; Yang, B.; Wu, H.; Wang, W.; Song, H.; Huang, B.; Zhu, N.; Bi, Y.; Ma, X.; Zhan, F.; Wang, L.; Hu, T.; Zhou, H.; Hu, Z.; Zhou, W.; Zhao, L.; Chen, J.; Meng, Y.; Wang, J.; Lin, Y.; Yuan, J.; Xie, Z.; Ma, J.; Liu, W.J.; Wang, D.; Xu, W.; Holmes, E.C.; Gao, G.F.; Wu, G.; Chen, W.; Shi, W.; Tan, W. Genomic characterisation and epidemiology of 2019 novel coronavirus: implications for virus 
origins and receptor binding. The Lancet 2020, 395, 565-574, https://doi.org/10.1016/S01406736(20)30251-8.

36. Chen Y.; Liu Q.; Guo D.; Emerging coronaviruses: genome structure, replication, and pathogenesis. J Med Virol. 2020, 92, 418-423, https://doi.org/10.1002/jmv.25681.

37. Keane, S.C.; Giedroc, D.P. Solution structure of mouse hepatitis virus (MHV) nsp3a and determinants of the interaction with MHV nucleocapsid (N) protein. $J$ Virol 2013, 87, 3502-3515, https://doi.org/10.1128/JVI.03112-12.

38. CDC COVID-19 Response Team. Coronavirus Disease 2019 in Children - United States, February 12-April 2, 2020. MMWR Morb Mortal Wkly Rep 2020, 69, http://dx.doi.org/10.15585/mmwr.mm6914e4.

39. Xu, L.; Liu, H.; Murray, B.P.; Callebaut, C.; Lee, M.S.; Hong, A.; Strickley, R.G.; Tsai, L.K.; Stray, K.M.; Wang, Y.; Rhodes, G.R.; Desai, M.C. Cobicistat (GS-9350): A Potent and Selective Inhibitor of Human CYP3A as a Novel Pharmacoenhancer. ACS Medicinal Chemistry Letters 2010, 1, 209-213, https://doi.org/10.1021/ml1000257.

40. Bartesaghi, A.; Merk, A.; Borgnia, M.J.; Milne, J.L.S.; Subramaniam, S. Prefusion structure of trimeric HIV1 envelope glycoprotein determined by cryo-electron microscopy. Nature Structural \& Molecular Biology 2013, 20, 1352-1357, http://dx.doi.org/10.1038/nsmb.2711.

41. Hofmann, H.; Hattermann, K.; Marzi, A.; Gramberg, T.; Geier, M.; Krumbiegel, M.; Kuate, S.; Überla, K.; Niedrig, M.; Pöhlmann, S. S Protein of Severe Acute Respiratory Syndrome-Associated Coronavirus Mediates Entry into Hepatoma Cell Lines and Is Targeted by Neutralizing Antibodies in Infected Patients. $J$ Virol 2004, 78, 6134-6142, http://dx.doi.org/10.1128/JVI.78.12.6134-6142.2004.

42. Walls, A.C.; Tortorici, M.A.; Bosch, B.-J.; Frenz, B.; Rottier, P.J.M.; DiMaio, F.; Rey, F.A.; Veesler, D. Cryo-electron microscopy structure of a coronavirus spike glycoprotein trimer. Nature 2016, 531, 114-117, http://dx.doi.org/10.1038/nature16988.

43. Kirchdoerfer, R.N.; Cottrell, C.A.; Wang, N.; Pallesen, J.; Yassine, H.M.; Turner, H.L.; Corbett, K.S.; Graham, B.S.; McLellan, J.S.; Ward, A.B. Pre-fusion structure of a human coronavirus spike protein. Nature 2016, 531, 118-121, http://dx.doi.org/10.1038/nature17200.

44. Lin, X.; Eddy, N.R.; Noel, J.K.; Whitford, P.C.; Wang, Q.; Ma, J.; Onuchic, J.N. Order and disorder control the functional rearrangement of influenza hemagglutinin. Proceedings of the National Academy of Sciences 2014, 111, 12049-12054, http://dx.doi.org/10.1073/pnas.1412849111.

45. Bosch, B.J.; Van Der Zee, R.; de Haan, C.A.M.; Rottier, P.J.M. The coronavirus spike protein is a class I virus fusion protein: structural and functional characterization of the fusion core complex. J. Virol. 2003, 77, 8801-8811, https://doi.org/10.1128/jvi.77.16.8801-8811.2003.

46. Zhu, H.; Wang, L.; Fang, C.; Peng, S.; Zhang, L.; Chang, G.; Xia, S.; Zhou, W. Clinical analysis of 10 neonates born to mothers with 2019-nCoV pneumonia. Translational Pediatrics 2020, 9, 51-60, https://doi.org/10.21037/tp.2020.02.06.

47. Cascella M, Rajnik M, Cuomo A, Dulebohn SC, Napoli RD: Features, Evaluation and Treatment Coronavirus (COVID-19). In: StatPearls. Publishing, Treasure Island, FL; 2020.

48. Hadi, A.; Kadhom, M.; Hairunisa, N.; Yousif, E.; Mohammed, S., A Review on COVID-19: Origin, Spread, Symptoms, Treatment, and Prevention, Biointerface Res. Appl. 10, 6 (2020) 7234 - 7242. https://doi.org/10.33263/BRIAC 106.723472

49. Wu, K.; Li, W.; Peng, G.; Li, F. Crystal structure of NL63 respiratory coronavirus receptor-binding domain complexed with its human receptor. Proc Natl Acad Sci USA 2009, 106, 19970-19974, https://doi.org/10.1073/pnas.0908837106.

50. Xu, Y.; Lou, Z.; Liu, Y.; Pang, H.; Tien, P.; Gao, G.F.; Rao, Z. Crystal structure of severe acute respiratory syndrome coronavirus spike protein fusion core. J Biol Chem 2004, 279, 49414-49419, http://dx.doi.org/10.1074/jbc.M408782200.

51. Ou, X.; Zheng, W.; Shan, Y.; Mu, Z.; Dominguez, S.R.; Holmes, K.V.; Qian, Z. Identification of the Fusion Peptide-Containing Region in Betacoronavirus Spike Glycoproteins. J Virol 2016, 90, 5586-5600, https://doi.org/10.1128/jvi.00015-16.

52. Sprang, G.; Silman, M. Posttraumatic Stress Disorder in Parents and Youth After Health-Related Disasters. Disaster Medicine and Public Health Preparedness 2013, 7, 105-110, https://doi.org/10.1017/dmp.2013.22.

53. Lu, L.; Liu, Q.; Zhu, Y.; Chan, K.-H.; Qin, L.; Li, Y.; Wang, Q.; Chan, J.F.-W.; Du, L.; Yu, F.; Ma, C.; Ye, S.; Yuen, K.-Y.; Zhang, R.; Jiang, S. Structure-based discovery of Middle East respiratory syndrome coronavirus fusion inhibitor. Nature Communications 2014, $5, \quad 3067$, http://dx.doi.org/10.1038/ncomms4067.

54. Huey, R.; Morris, G. AutoDock Tools. The Scripps Research Institute: La Jolla, CA, USA, 2003.

55. Duquerroy, S.; Vigouroux, A.; Rottier, P.J.; Rey, F.A.; Bosch, B.J. Central ions and lateral asparagine/glutamine zippers stabilize the post-fusion hairpin conformation of the SARS coronavirus spike glycoprotein. Virology 2005, 335, 276-285, https://doi.org/10.1016/j.virol.2005.02.022.

56. Gao, J.; Lu, G.; Qi, J.; Li, Y.; Wu, Y.; Deng, Y.; Geng, H.; Li, H.; Wang, Q.; Xiao, H.; Tan, W.; Yan, J.; Gao, G.F. Structure of the Fusion Core and Inhibition of Fusion by a Heptad Repeat Peptide Derived from the S Protein of Middle East Respiratory Syndrome Coronavirus. J Virol 2013, 87, 13134-13140, http://dx.doi.org/10.1128/JVI.02433-13. 
57. Wei, M.; Yuan, J.; Liu, Y.; Fu, T.; Yu, X.; Zhang, Z.-J. Novel Coronavirus Infection in Hospitalized Infants Under 1 Year of Age in China. JAMA 2020, 323, 1313-1314, https://doi.org/10.1001/jama.2020.2131.

58. Monajjemi, M. Graphene/(h-BN)n/X-doped raphene as anode material in lithium ion batteries $(\mathrm{X}=\mathrm{Li}, \mathrm{Be}$, B AND N). Macedonian Journal of Chemistry and Chemical Engineering 2017, 36, 101-118, http://dx.doi.org/10.20450/mjcce.2017.1134.

59. Jalilian, H.; Monajjemi, M. Capacitor simulation including of $\mathrm{X}$-doped graphene $(\mathrm{X}=\mathrm{Li}, \mathrm{Be}, \mathrm{B})$ as two electrodes and (h-BN)m (m = 1-4) as the insulator. Japanese Journal of Applied Physics 2015, 54, 0851017.

60. Ardalan, T.; Ardalan, P.; Monajjemi, M. Nano theoretical study of a C 16 cluster as a novel material for vitamin C carrier. Fullerenes Nanotubes and Carbon Nanostructures 2014, 22, 687-708, https://doi.org/10.1080/1536383X.2012.717561.

61. Shang, J.; Ye, G.; Shi, K.; Wan, Y.S.; Aihara, H.; Li, F. Structural basis for resptor recognition by novel coronavirus from wuhan. Structural biology virology 2020, 03-04, https://doi.org/10.21203/rs.2.24749/v1.

62. Xia, S.; Yan, L.; Xu, W.; Agrawal, A.S.; Algaissi, A.; Tseng, C.K.; Wang, Q.; Du, L.; Tan, W.; Wilson, I.A.; Jiang, S.; Yang, B.; Lu, L. A pan-coronavirus fusion inhibitor targeting the HR1 domain of human coronavirus spike. Sci Adv 2019, 5, eaav4580-eaav4580, https://doi.org/doi:10.1126/sciadv.aav4580.

63. Rothe, C.; Schunk, M.; Sothmann, P.; Bretzel, G.; Froeschl, G.; Wallrauch, C.; Zimmer, T.; Thiel, V.; Janke, C.; Guggemos, W.; Seilmaier, M.; Drosten, C.; Vollmar, P.; Zwirglmaier, K.; Zange, S.; Wölfel, R.; Hoelscher, M. Transmission of 2019-nCoV Infection from an Asymptomatic Contact in Germany. New England Journal of Medicine 2020, 382, 970-971, https://doi.org/10.1056/NEJMc2001468.

64. Lu, X.; Zhang, L.; Du, H.; Zhang, J.; Li, Y.Y.; Qu, J.; Zhang, W.; Wang, Y.; Bao, S.; Li, Y.; Wu, C.; Liu, H.; Liu, D.; Shao, J.; Peng, X.; Yang, Y.; Liu, Z.; Xiang, Y.; Zhang, F.; Silva, R.M.; Pinkerton, K.E.; Shen, K.; Xiao, H.; Xu, S.; Wong, G.W.K. SARS-CoV-2 Infection in Children. New England Journal of Medicine 2020, 382, 1663-1665, https://doi.org/10.1056/NEJMc2005073.

65. Hafeez, A.; Ahmad, S.; Siddqui, S.A.; Ahmad, M.; Mishra, S. A Review of COVID-19 (Coronavirus Disease-2019) Diagnosis, Treatments and Prevention. EJMO 2020, 4, 116-125, https://dx.doi.org/10.14744/ejmo.2020.90853.

66. Jin, Y.H.; Cai, L.; Cheng, Z.S.; Cheng, H.; Deng, T.; Fan, Y.P.; Fang, C.; Huang, D.; Huang, L.Q.; Huang, Q.; Han, Y.; Hu, B.; Hu, F.; Li, B.-H.; Li, Y.R.; Liang, K.; Lin, L.K.; Luo, L.-S.; Ma, J.; Ma, L.L.; Peng, Z.Y.; Pan, Y.B.; Pan, Z.Y.; Ren, X.Q.; Sun, H.M.; Wang, Y.; Wang, Y.Y.; Weng, H.; Wei, C.J.; Wu, D.F.; Xia, J.; Xiong, Y.; Xu, H.B.; Yao, X.M.; Yuan, Y.F.; Ye, T.S.; Zhang, X.C.; Zhang, Y.W.; Zhang, Y.G.; Zhang, H.M.; Zhao, Y.; Zhao, M.J.; Zi, H.; Zeng, X.T.; Wang, Y.Y.; Wang, X.H. Management, f.t.Z.H.o.W.U.N.C.; Research Team, E.-B.M.C.o.C.I.E.; Promotive Association for, M.; Health, C. A rapid advice guideline for the diagnosis and treatment of 2019 novel coronavirus (2019-nCoV) infected pneumonia (standard version). Military Medical Research 2020, 7, https://doi.org/10.1186/s40779-020-0233-6.

67. Sun, P.; Lu, X.; Xu, C.; Sun, W.; Pan, B. Understanding of COVID-19 based on current evidence. Journal of Medical Virology 2020, 92, 548-551, https://doi.org/10.1002/jmv.25722.

68. Sabir, D.K.; Sidiq, K.R.; Ali, S.M. Current Speculations on the Low Incidence Rate of the COVID-19 among Children. International Journal of School Health 2020, 7, 61-62.

69. Ahmadpoor, P.; Rostaing, L. Why the immune system fails to mount an adaptive immune response to a COVID-19 infection. Transplant International 2020, 1-2, https://doi.org/10.1111/tri.13611.

70. Li, F. Receptor Recognition Mechanisms of Coronaviruses: a Decade of Structural Studies. J Virol 2015, 89, 1954-1964, http://dx.doi.org/10.1128/JVI.02615-14.

71. Rudolph, M.E.; McArthur, M.A.; Barnes, R.S.; Magder, L.S.; Chen, W.H.; Sztein, M.B. Differences Between Pediatric and Adult T Cell Responses to In Vitro Staphylococcal Enterotoxin B Stimulation. Frontiers in Immunology 2018, 9, https://doi.org/10.3389/fimmu.2018.00498.

72. Li, W.; Zhang, C.; Sui, J.; Kuhn, J.H.; Moore, M.J.; Luo, S.; Wong, S.-K.; Huang, I.C.; Xu, K.; Vasilieva, N.; Murakami, A.; He, Y.; Marasco, W.A.; Guan, Y.; Choe, H.; Farzan, M. Receptor and viral determinants of SARS-coronavirus adaptation to human ACE2. The EMBO Journal 2005, 24, 1634-1643, http://dx.doi.org/10.1038/sj.emboj.7600640.

73. Phelan, A.L.; Katz, R.; Gostin, L.O. The Novel Coronavirus Originating in Wuhan, China: Challenges for Global Health Governance. JAMA 2020, 323, 709-710, https://doi.org/10.1001/jama.2020.1097.

74. Gorbalenya, A.E.; Baker, S.C.; Baric, R.S.; de Groot, R.J.; Drosten, C.; Gulyaeva, A.A.; Haagmans, B.L.; Lauber, C.; Leontovich, A.M.; Neuman, B.W.; Penzar, D.; Perlman, S.; Poon, L.L.M.; Samborskiy, D.; Sidorov, I.A.; Sola, I.; Ziebuhr, J. \&lt;em\&gt;Severe acute respiratory syndrome-related coronavirus\&lt;/em\&gt;: The species and its viruses - a statement of the Coronavirus Study Group. bioRxiv 2020, https://doi.org/10.1101/2020.02.07.937862.

75. Bonavia, A.; Zelus, B.D.; Wentworth, D.E.; Talbot, P.J.; Holmes, K.V. Identification of a receptor-binding domain of the spike glycoprotein of human coronavirus HCoV-229E. J Virol 2003, 77, 2530-2538, http://dx.doi.org/10.1128/JVI.77.4.2530-2538.2003.

76. Russell, C.D.; Millar, J.E.; Baillie, J.K. Clinical evidence does not support corticosteroid treatment for 2019nCoV lung injury. The Lancet 2020, 395, 473-475, https://doi.org/10.1016/S0140-6736(20)30317-2. 
77. Zhao, J.P.; Hu, Y.; Du, R.H.; Chen, Z.S.; Jin, Y.; Zhou, M.; Zhang, J.; Qu, J.M.; Cao, B. [Expert consensus on the use of corticosteroid in patients with 2019-nCoV pneumonia]. Zhonghua Jie He He Hu Xi Za Zhi 2020, 43, 183-184, https://doi.org/10.3760/cma.j.issn.1001-0939.2020.0007.

78. Sidiq, K.R.; Sabir, D.K.; Ali, S.M.; Kodzius, R. Does Early Childhood Vaccination Protect Against COVID19? Frontiers in Molecular Biosciences 2020, 7, https://doi.org/10.3389/fmolb.2020.00120.

79. Sanders, J.M.; Monogue, M.L.; Jodlowski, T.Z.; Cutrell, J.B. Pharmacologic Treatments for Coronavirus Disease 2019 (COVID-19): A Review. JAMA 2020, 323, 1824-1836, https://doi.org/10.1001/jama.2020.6019.

80. Grein, J.; Ohmagari, N.; Shin, D.; Diaz, G.; Asperges, E.; Castagna, A.; Feldt, T.; Green, G.; Green, M.L.; Lescure, F.-X.; Nicastri, E.; Oda, R.; Yo, K.; Quiros-Roldan, E.; Studemeister, A.; Redinski, J.; Ahmed, S.; Bernett, J.; Chelliah, D.; Chen, D.; Chihara, S.; Cohen, S.H.; Cunningham, J.; D’Arminio Monforte, A.; Ismail, S.; Kato, H.; Lapadula, G.; L’Her, E.; Maeno, T.; Majumder, S.; Massari, M.; Mora-Rillo, M.; Mutoh, Y.; Nguyen, D.; Verweij, E.; Zoufaly, A.; Osinusi, A.O.; DeZure, A.; Zhao, Y.; Zhong, L.; Chokkalingam, A.; Elboudwarej, E.; Telep, L.; Timbs, L.; Henne, I.; Sellers, S.; Cao, H.; Tan, S.K.; Winterbourne, L.; Desai, P.; Mera, R.; Gaggar, A.; Myers, R.P.; Brainard, D.M.; Childs, R.; Flanigan, T. Compassionate Use of Remdesivir for Patients with Severe Covid-19. New England Journal of Medicine 2020, 382, 2327-2336, https://doi.org/10.1056/NEJMoa2007016.

81. Shen, C.; Wang, Z.; Zhao, F.; Yang, Y.; Li, J.; Yuan, J.; Wang, F.; Li, D.; Yang, M.; Xing, L.; Wei, J.; Xiao, H.; Yang, Y.; Qu, J.; Qing, L.; Chen, L.; Xu, Z.; Peng, L.; Li, Y.; Zheng, H.; Chen, F.; Huang, K.; Jiang, Y.; Liu, D.; Zhang, Z.; Liu, Y.; Liu, L. Treatment of 5 Critically Ill Patients With COVID-19 With Convalescent Plasma. JAMA 2020, 323, 1582-1589, https://doi.org/10.1001/jama.2020.4783.

82. Li, J.; Li, J.; Xie, X.; Cai, X.; Huang, J.; Tian, X.; Zhu, H. Game consumption and the 2019 novel coronavirus. The Lancet Infectious Diseases 2020, 20, 275-276, https://doi.org/10.1016/S14733099(20)30063-3.

83. Huang, C.; Wang, Y.; Li, X.; Ren, L.; Zhao, J.; Hu, Y.; Zhang, L.; Fan, G.; Xu, J.; Gu, X.; Cheng, Z.; Yu, T.; Xia, J.; Wei, Y.; Wu, W.; Xie, X.; Yin, W.; Li, H.; Liu, M.; Xiao, Y.; Gao, H.; Guo, L.; Xie, J.; Wang, G.; Jiang, R.; Gao, Z.; Jin, Q.; Wang, J.; Cao, B. Clinical features of patients infected with 2019 novel coronavirus in Wuhan, China. The Lancet 2020, 395, 497-506, https://doi.org/10.1016/S01406736(20)30183-5.

84. Al-mashhadani, M.; Alsayed, R.; Hussain, Z.; Salih, N.; Yousif E. An Overview of Possible Therapeutic Approaches Against Novel Coronavirus Disease 2019 Pandemic. Al-Nahrain Journal of Science 2020, 3, 611.

85. Chen, N.; Zhou, M.; Dong, X.; Qu, J.; Gong, F.; Han, Y.; Qiu, Y.; Wang, J.; Liu, Y.; Wei, Y.; Xia, J.a.; Yu, T.; Zhang, X.; Zhang, L. Epidemiological and clinical characteristics of 99 cases of 2019 novel coronavirus pneumonia in Wuhan, China: a descriptive study. The Lancet 2020, 395, 507-513, https://doi.org/10.1016/S0140-6736(20)30211-7.

86. Yang, X.; Yu, Y.; Xu, J.; Shu, H.; Xia, J.a.; Liu, H.; Wu, Y.; Zhang, L.; Yu, Z.; Fang, M.; Yu, T.; Wang, Y.; Pan, S.; Zou, X.; Yuan, S.; Shang, Y. Clinical course and outcomes of critically ill patients with SARS-CoV2 pneumonia in Wuhan, China: a single-centered, retrospective, observational study. The Lancet Respiratory Medicine 2020, 8, 475-481, https://doi.org/10.1016/S2213-2600(20)30079-5.

87. Zou, L.; Ruan, F.; Huang, M.; Liang, L.; Huang, H.; Hong, Z.; Yu, J.; Kang, M.; Song, Y.; Xia, J.; Guo, Q.; Song, T.; He, J.; Yen, H.-L.; Peiris, M.; Wu, J. SARS-CoV-2 Viral Load in Upper Respiratory Specimens of Infected Patients. New England Journal of Medicine 2020, 382, 1177-1179, https://doi.org/10.1056/NEJMc2001737.

88. Qiu, H.; Tong, Z.; Ma, P.; Hu, M.; Peng, Z.; Wu, W.; Du, B.; China Critical Care Clinical Trials, G. Intensive care during the coronavirus epidemic. Intensive Care Medicine 2020, 46, 576578, https://doi.org/10.1007/s00134-020-05966-y.

89. Wang, D.; Hu, B.; Hu, C.; Zhu, F.; Liu, X.; Zhang, J.; Wang, B.; Xiang, H.; Cheng, Z.; Xiong, Y.; Zhao, Y.; Li, Y.; Wang, X.; Peng, Z. Clinical Characteristics of 138 Hospitalized Patients With 2019 Novel Coronavirus-Infected Pneumonia in Wuhan, China. JAMA 2020, 323, 1061-1069, https://doi.org/10.1001/jama.2020.1585.

90. Wang, Z.; Chen, X.; Lu, Y.; Chen, F.; Zhang, W. Clinical characteristics and therapeutic procedure for four cases with 2019 novel coronavirus pneumonia receiving combined Chinese and Western medicine treatment. BioScience Trends 2020, 14, 64-68, https://doi.org/10.5582/bst.2020.01030.

91. Arabi, Y.M.; Fowler, R.; Hayden, F.G. Critical care management of adults with community-acquired severe respiratory viral infection. Intensive Care Medicine 2020, 46, 315-328, https://doi.org/10.1007/s00134-02005943-5.

92. Sun, P.; Qie, S.; Liu, Z.; Ren, J.; Li, K.; Xi, J. Clinical characteristics of hospitalized patients with SARSCoV-2 infection: A single arm meta-analysis. Journal of Medical Virology 2020, 92, 612617, https://doi.org/10.1002/jmv.25735.

93. Wang, Y.; Fan, G.; Salam, A.; Horby, P.; Hayden, F.G.; Chen, C.; Pan, J.; Zheng, J.; Lu, B.; Guo, L.; Wang, C.; Cao, B.; Community-Acquired Pneumonia China, N. Comparative Effectiveness of Combined Favipiravir and Oseltamivir Therapy Versus Oseltamivir Monotherapy in Critically Ill Patients With 
Influenza Virus Infection. The Journal of Infectious Diseases 2019, 221, 16881698, https://doi.org/10.1093/infdis/jiz656.

94. Dong, Y.; Mo, X.; Hu, Y.; Qi, X.; Jiang, F.; Jiang, Z.; Tong, S. Epidemiological Characteristics of 2143 Pediatric Patients With 2019 Coronavirus Disease in China. Pediatrics 2020, 145, https://doi.org/10.1542/peds.2020-0702.

95. Gordon, C.J.; Tchesnokov, E.P.; Feng, J.Y.; Porter, D.P.; Götte, M. The antiviral compound remdesivir potently inhibits RNA-dependent RNA polymerase from Middle East respiratory syndrome coronavirus. $J$ Biol Chem 2020, 295, 4773-4779, https://doi.org/10.1074/jbc.AC120.013056.

96. de Wit, E.; Feldmann, F.; Cronin, J.; Jordan, R.; Okumura, A.; Thomas, T.; Scott, D.; Cihlar, T.; Feldmann, H. Prophylactic and therapeutic remdesivir (GS-5734) treatment in the rhesus macaque model of MERS$\mathrm{CoV}$ infection. Proceedings of the National Academy of Sciences 2020, 117, 67716776, https://doi.org/10.1073/pnas.1922083117.

97. Lu, H. Drug treatment options for the 2019-new coronavirus (2019-nCoV). BioScience Trends 2020, 14, 6971, https://doi.org/10.5582/bst.2020.01020.

98. Centers for Disease Control and Prevention, National Center for Immunization and Respiratory Diseases (NCIRD), Division of Viral Diseases. Coronavirus Disease 2019 (COVID-19); 2020.

99. Yao, X.; Ye, F.; Zhang, M.; Cui, C.; Huang, B.; Niu, P.; Liu, X.; Zhao, L.; Dong, E.; Song, C.; Zhan, S.; Lu, R.; Li, H.; Tan, W.; Liu, D. In Vitro Antiviral Activity and Projection of Optimized Dosing Design of Hydroxychloroquine for the Treatment of Severe Acute Respiratory Syndrome Coronavirus 2 (SARS-CoV2). Clinical Infectious Diseases 2020, https://doi.org/10.1093/cid/ciaa237.

100. Chen, L.; Xiong, J.; Bao, L.; Shi, Y. Convalescent plasma as a potential therapy for COVID-19. The Lancet Infectious Diseases 2020, 20, 398-400, https://doi.org/10.1016/s1473-3099(20)30141-9.

101. Alhazzani, W.; Møller, M.H.; Arabi, Y.M.; Loeb, M.; Gong, M.N.; Fan, E.; Oczkowski, S.; Levy, M.M.; Derde, L.; Dzierba, A.; Du, B.; Aboodi, M.; Wunsch, H.; Cecconi, M.; Koh, Y.; Chertow, D.S.; Maitland, K.; Alshamsi, F.; Belley-Cote, E.; Greco, M.; Laundy, M.; Morgan, J.S.; Kesecioglu, J.; McGeer, A.; Mermel, L.; Mammen, M.J.; Alexander, P.E.; Arrington, A.; Centofanti, J.E.; Citerio, G.; Baw, B.; Memish, Z.A.; Hammond, N.; Hayden, F.G.; Evans, L.; Rhodes, A. Surviving Sepsis Campaign: guidelines on the management of critically ill adults with Coronavirus Disease 2019 (COVID-19). Intensive Care Medicine 2020, 46, 854-887, https://doi.org/10.1007/s00134-020-06022-5.

102. MacLaren, G.; Fisher, D.; Brodie, D. Preparing for the Most Critically Ill Patients With COVID-19: The Potential Role of Extracorporeal Membrane Oxygenation. JAMA 2020, 323, 12451246, https://doi.org/10.1001/jama.2020.2342.

103. Poston, J.T.; Patel, B.K.; Davis, A.M. Management of Critically Ill Adults With COVID-19. JAMA 2020, 323, 1839-1841, https://doi.org/10.1001/jama.2020.4914. 